\title{
Evolution of the microstructure, chemical composition and magnetic behaviour during the synthesis of alkanethiol-capped gold nanoparticles.
}

E. Guerrero', T.C. Rojas ${ }^{1}$, M. Multigner ${ }^{2}$, P. Crespo ${ }^{2}$, M.A. Muñoz-Márquez, M.A. García, A. Hernando ${ }^{2}$, A. Fernández ${ }^{1^{*}}$

${ }^{1}$ Instituto de Ciencia de Materiales de Sevilla CSIC-Univ.Sevilla, Américo Vespucio 49, 41092 Sevilla, Spain.

${ }^{2}$ Instituto de Magnetismo Aplicado (RENFE-UCM-CSIC), P.O. Box 155, 28230, Las Rozas, Madrid, Spain and Departamento de Física de Materiales, Universidad Complutense de Madrid, 28040 Madrid, Spain.

* Corresponding author: Tel.: +34 954489531; fax: +34 954460665.

E-mail address: asuncion@,icmse.csic.es

Keywords: gold nanoparticles, microstructure, magnetic behaviour

\begin{abstract}
In the present paper we show an exhaustive microstructural characterisation of thiolcapped gold nanoparticles (NPs) with two different average particle sizes. These samples are compared to the polymer-like $\mathrm{Au}(\mathrm{I})$ phase formed as a precursor during the synthesis of the thiol-capped gold NPs. The set of analysed samples shows different microstructures at the nanoscale with different proportion of $\mathrm{Au}$ atoms bonded either to $\mathrm{S}$ or to $\mathrm{Au}$ atoms. It has been experimentally shown that the presence of a ferromagnetic-like behaviour is associated to the formation of NPs with simultaneous presence of $\mathrm{Au}-\mathrm{Au}$ and $\mathrm{Au}-\mathrm{S}$ bonds. In order to explain such magnetic behaviour a possible model is proposed based on the spin-orbit coupling so that localised charges and/or spins (Au-S bonds) can trap conduction electrons ( $\mathrm{Au}-\mathrm{Au}$ bonds) in orbits.
\end{abstract}




\section{Introduction}

Metallic nanoparticles (NPs) are extensively studied since they exhibit novel electronic, optical and magnetic properties. In particular, the study of magnetism in clusters of palladium and gold, and in general $4 d$ elements, has attracted special attention as these elements are non-magnetic as bulk materials [1-7]. Alkanethiol-capped gold NPs [4], as well as self-assembled alkanethiol monolayers on gold surfaces [3], have shown a permanent magnetism which currently is not yet understood [4, 8-11]. Recently, Hernando et al. $[9,11]$ have proposed spin-orbit coupling as the origin of orbital ferromagnetism in these systems. New experiments are still needed to correlate the microstructure and the magnetic behaviour of the alkanethiol-capped gold NPs.

The original method proposed by Brust et al. [12] to synthesize the alkanethiolate gold NPs is a two-step process $[13,14]$ :

$$
\begin{gathered}
\left.\mathrm{AuCl}_{4}{ }^{-} \text {(toluene }\right)+\mathrm{RSH} \rightarrow\left(-\mathrm{Au}^{\mathrm{I} S R}-\right)_{\mathrm{n}}(\text { polymer }) \\
\left(-\mathrm{Au}^{\mathrm{I} S R}-\right)_{\mathrm{n}}+\mathrm{BH}_{4}{ }^{-} \rightarrow \mathrm{Au}_{\mathrm{x}}(\mathrm{SR})_{\mathrm{y}} / 2 /
\end{gathered}
$$

In the first step, $\mathrm{Au}(\mathrm{III})$ is reduced to $\mathrm{Au}(\mathrm{I})$ by oxidizing the thiols to disulfides. In non polar solvents, the $\mathrm{Au}(\mathrm{SR})$ species aggregate forming a polymeric phase [13,14]. In the second step, the addition of sodium borohydride leads to reduction of all gold species in order to form the gold clusters surrounded by thiolates $[13,14]$.

In the present paper we have isolated the intermediate AuSR(polymeric) phase, as well as different gold NPs obtained under different experimental conditions. In all these samples we have carefully carried out a study of the microstructure, the chemical composition and the magnetic behaviour in order to understand the nature of the nanostructures responsible of the anomalous ferromagnetic behaviour.

\section{Experimental}

\section{Sample preparation}

The alkanethiolate-capped gold NPs have been obtained from a liquid-liquid phase reduction at room temperature following the Burst method [12]; the Au:thiol molar ratio has been modified in order to produce different samples (Au-SR1 and Au-SR2). First, $\mathrm{Au}(\mathrm{III})$ is transferred from an aqueous solution containing $\mathrm{AuCl}_{4} \mathrm{H}$ (FLUKA 99\%, $0.0064 \mathrm{~g}$ in $6.4 \mathrm{ml}$ of milli-Q water) to degassed and dried toluene, using 
tetraoctylammonium bromide (Aldrich 98\%, $0.112 \mathrm{~g}$ in $20 \mathrm{ml}$ of toluene) as the phasetransfer agent. The mixture is strongly stirred for 10 minutes. Once the aqueous phase is removed, $0.1 \mathrm{ml}$ (for the Au-SR2 sample) or $0.05 \mathrm{ml}$ (for Au-SR1 sample) of dodecanethiol (Aldrich 98.5\%) is added to the organic phase under strong stirring for 5 minutes. Then the Au-SR polymeric precursor is reduced, using an aqueous solution of $\mathrm{NaBH}_{4}$ (Aldrich 99\%, $0.1 \mathrm{~g}$ in $6 \mathrm{ml}$ of milli-Q $\mathrm{H}_{2} \mathrm{O}$ ) as reducing agent. The presence of dodecanethiol leads to the formation of Au-S bonds which isolate the metal clusters preventing from cluster agglomeration. Subsequently, the organic phase was separated from the aqueous phase and the toluene was removed under reduced pressure by means of a rotary evaporator. Finally, Au NPs were precipitated with ethanol, filtered, washed and dried. The Au:thiol ratio is one of the nanocluster size-controlling factors [15]. With the aim to obtain gold nanoparticles with different average sizes, reactions have been carried out for Au:thiol molar ratios ranging from 1:2 (sample referred as Au-SR2) to 1:1 (sample referred as Au-SR1 sample).

The intermediate Au-SR polymeric precursor (sample referred as Polymer) was isolated for the 1:2 Au:thiol ratio by removing the toluene solvent, just before the reduction agent $\mathrm{NaBH}_{4}$ is added. After precipitation with ethanol, filtering, washing and drying, the sample was stored in a desiccator. After ageing in storage conditions for several weeks, the sample named Polymer-aged was also investigated.

All the reactions during preparation of the different samples were carried out under $\mathrm{N}_{2}$ atmosphere to minimize oxidation. Ulterior washing, drying, storage and transfer to SQUID, TEM, UV-Vis, XPS and EXAFS were carried out in ambient air atmosphere. The samples were stored in a desiccator.

\section{Sample characterisation}

The chemical composition of the samples has been evaluated by ICP (Inductively Coupled Plasma-atomic emission spectrometry) analysis, elemental chemical analysis and energy dispersive X-ray analysis (EDX) at the transmission electron microscope.

TEM analysis was carried out in a Philips CM200 microscope working at $200 \mathrm{kV}$. Particle size distribution histograms were measured using an image analyser. 
X-ray absorption spectra (XAS) for the Au samples were recorded in transmission mode at the BM29 beam line in the ESRF storage ring in very thin self-supported pressed pellets. Spectra were recorded at the $\mathrm{Au} \mathrm{L}_{2}$ - and $\mathrm{L}_{3}$-edge at $13.734 \mathrm{keV}$ and $11.919 \mathrm{keV}$ respectively. The EXAFS oscillations were quantitatively analyzed with the software package developed by Bonin et al [16]. The coordination numbers $(\mathrm{N})$, bond distances (R) and Debye-Waller factors $(\sigma)$ were extracted by a least-squares fitting procedure that uses the theoretical phases and amplitudes proposed by Rehr and coworkers [17]. These parameters have been previously compared with $\mathrm{Au}$ foil and $\mathrm{Au}_{2} \mathrm{~S}$ (Aldrich 99.9\%) reference samples.

UV-Vis absorption spectra were recorded in transmission mode. In this experiment, the gold NPs were dispersed in aqueous solutions $(1 \mathrm{mg} / \mathrm{ml})$. The spectra were recorded in the range $200-850 \mathrm{~nm}$ with a Shimadzu UV-2102 PC spectrometer.

XPS analysis of the $\mathrm{Au} 4 f, \mathrm{Au} 5 d, \mathrm{Au} 6 s, \mathrm{~S} 2 p$, and $\mathrm{C} 1 s$ photoelectron spectra was performed using a SPECS Phoibos HSA3500 100 spectrometer; which has a five channel hemispheric analyser for photoelectron registration. This incorporates a nonmonochromised Al/Mg twin anode SPECS XR-50 x-ray source. The radiation used in this experiment is the $\mathrm{Mg} K \alpha(h v=1253.6 \mathrm{eV})$. The analyser was operated in constant energy analyser mode at a pass energy of $20 \mathrm{eV}$ and with $0.8 \mathrm{~mm}$ slits.

Since the NPs samples are not good electrical conductors, an external binding energy (BE) calibration standard such as gold or silver is of no use. The NPs samples will suffer charging effects. Therefore, a calibration standard within the sample is needed. The carbon peak $\mathrm{C} 1 s$ is probably the best choice; no strong bonding between contamination carbon and gold is expected.

Curve fitting of the photoelectron peaks has been performed using a Shirley background and Voigt functions. This fit allowed us to determine the elemental peak areas which were weighted by the photoionisation cross sections calculated by Yeh and Lindau [19]; once the corrected areas were known then, elemental quantification was possible Table 1. The data fitting process was carried out using a least squares routine implemented in a computer program [20].

Magnetic measurements have been performed using a Quantum Design SQUID magnetometer. The sample holder used is adhesive kapton stocked to a quartz tube. 
Diamagnetic contribution corresponding to the sample holder system has been previously measured and subtracted from total magnetization.

\section{Results and Discussions}

\section{Microstructural and chemical characterisation of the samples}

In Table 1, the chemical compositions of the three samples are summarized as determined by ICP ( $\mathrm{Au}$ and Fe content) and elemental chemical analysis $(\mathrm{C}, \mathrm{H}, \mathrm{S}$ content). Fe contamination in the samples has its origin on Fe impurities that chemicals and solvents can contain at the level of ppm. These impurities lead to some amount of dispersed $\mathrm{Fe}$ atoms in the samples. Au:S atomic ratios of 3.1, 2.0 and 1.0 were respectively found for the Au-SR1, Au-SR2 and Polymer samples. These results are congruent with the expected values for small nanoparticles (below $3.0 \mathrm{~nm}$ in diameter) and also for the polymeric $\mathrm{Au}(\mathrm{I})$ phase were a 1:1 $\mathrm{Au}$ to $\mathrm{S}$ atomic ratio has been proposed in previous investigations $[10,11]$.

Figure 1 presents the TEM analysis for the intermediate polymeric precursor under different conditions. In Fig. 1a we show the images recorded by short exposition of the samples to the e-beam. The images are congruent with a polymeric-like phase and the electron diffraction pattern shows the diffuse diffraction rings of an almost amorphous phase. If the sample is exposed to the TEM electron beam for a long time, then gold nanoparticles will be formed as we can see in Fig.1b. In this case, the electron diffraction pattern is already showing the rings corresponding to Au nanocrystals; an average particle diameter of $1.5 \mathrm{~nm}$ has been determined for this sample. The fact that the obtained gold NPs show a certain alignment along preferential directions is also of interest. This could be due to the microstructure of the polymeric phase which shows a column-like shape when looked at scanning electron microscope.

The Polymer sample is white in colour after preparation. After several weeks of storage in a desiccator (even protected from external light sources) the sample turns to a brownish coloured powder. The TEM micrograph obtained from the aged polymer sample is shown in Fig.1c. Big gold nanoparticles were formed indicating that the sample is not stable and degrades with time. The electron diffraction pattern is now showing well defined rings with visible points from the gold crystals in the diameter 
range $5-30 \mathrm{~nm}$. Some smaller crystals, similar to the ones obtained in Fig. $1 \mathrm{~b}$, are also seen in the micrograph. However, they could have also been produced inside the TEM microscope during e-beam irradiation.

For the Au-SR1 and Au-SR2 samples, the TEM micrograph included in Figs. 2a and 2b show the small gold nanoparticles with average particle sizes of 2.2 and $2.0 \mathrm{~nm}$ respectively. The slight decrease in average particle diameter for the Au-SR2 is the expected behaviour when decreasing Au:thiol molar ratio during the synthesis process. Electron diffraction patterns for these samples are similar to the ones showed in Fig.1b. Table 1 also shows the calculated total number of $\mathrm{Au}$ atoms and the percentage of atoms located at the surface for a pure gold cluster of the corresponding average particle size.

There is a significant interplay between the structure and the electronic and magnetic properties of the gold NPs. In fact, we have carried out a XAS analysis of the samples considering both the XANES and the EXAFS region of the spectra. Fig.3 shows the normalised $\mathrm{Au} \mathrm{L}_{2}$-edge XANES of the three samples compared to both a gold metal foil and a gold sulphide $\left(\mathrm{Au}_{2} \mathrm{~S}\right)$ reference sample. The more significant observation is the intensity of the resonance at the threshold (white line) associated with a $2 p_{3 / 2,1 / 2}$ to $5 d_{5 / 2,3 / 2}$ dipole transition which is giving the densities of unoccupied $d$ states at the Fermi level [21]. A more intense white line relative to that of Au metal indicates an increase in $d$ hole population ( $d$ charge depletion in the NPs) just above the Fermi level. Given that $\mathrm{S}$ is more electronegative than $\mathrm{Au}$, it is expected an important charge transfer from $\mathrm{Au}$ to $\mathrm{S}$ for the gold sulphide reference sample: this is observed in Fig.3a. The formally described Polymer sample is also showing a similar white line intensity, as expected for the $\mathrm{Au}(\mathrm{I})$ formulation of this sample. The total dispersion of the gold atoms bonded to sulphur in the polymer phase is leading, also in this sample, to a maximum value of the hole density at the gold atom. By contrast, the Au-SR1 and Au-SR2 NPs show a decrease of the white line intensity which is closer to the value expected for the bulk gold. In any case, the nanoparticles have slightly higher white line intensity than pure gold (see Fig.3b), due to the surface modification of the gold atoms through Au-S bonds. In fact, the NPs show an increase in the hold density compared to the $d$-hole population for Au metal where this density has its origin in the $s-d$ hybridization. 
Fig.4 is showing the modulus of the Fourier transform of EXAFS oscillations at the Au $\mathrm{L}_{3}$-edge ( $\mathrm{k}$ weighted, $\mathrm{k}$ space range of 2.8-13.2 $\AA^{-1}$ without phase corrections) for the $\mathrm{Au}-\mathrm{SR} 2, \mathrm{Au}-\mathrm{SR} 1$ and Polymer samples as compared with bulk gold (Au foil) and $\mathrm{Au}_{2} \mathrm{~S}$ reference samples. The formation of a network of -Au-S-Au-S- bonds is clearly observed in the Fourier transform of the Polymer sample where the Au-S first coordination shell is the predominant feature observed in the FT curve. For the NPs, in addition to the $\mathrm{Au}-\mathrm{S}$ coordination shell, also the typical $\mathrm{Au}-\mathrm{Au}$ coordination peaks are presented. For the Au-SR2 sample, with the smallest average particle diameter, it is observed an increase in the ratio of $\mathrm{Au}-\mathrm{S}$ to $\mathrm{Au}-\mathrm{Au}$ bonds in comparison to the same ratio for the larger NPs from the Au-SR1 sample. This effect is the expected one for the increase of surface to bulk gold atoms ratio and for the decrease in Au:S atomic ratio for the smaller nanoparticles.

The quantification of these observations is shown in Fig.5 and Table 2 where the EXAFS fitting analysis has been summarized. The following structural parameters were determined: coordination numbers $(\mathrm{N})$, bond distances $(\mathrm{R})$ and Debye Waller factors $(\sigma)$. Fig. 5 shows experimental and fitted data for EXAFS oscillations and radial distribution functions for the three investigated samples. For the Au-SR1 and Au-SR2 samples, the $\mathrm{Au}-\mathrm{Au}$ and $\mathrm{Au}-\mathrm{S}$ coordination numbers are congruent with the decrease in particle size together with an increase in the number of surface gold atoms bonded to sulphur. In addition, Au-Au bond lengths of 2.80 and $2.77 \AA$ have been estimated for these samples which are shorter that the one evaluated for the gold foil ( $2.85 \AA$ ). This indicates a lattice contraction already reported for small gold clusters [23]. These results are also in agreement with the measured white line intensity at the $\mathrm{Au} \mathrm{L}_{2}$ threshold, where lattice contraction effects are compensated by Au-S charge transfer effects. The latter results in a $d$-band hole density for the NPs only slightly higher than the one for bulk gold. The $\mathrm{Au}(\mathrm{I})$ polymeric sample shows $\mathrm{Au}-\mathrm{S}$ distances and coordination numbers congruent with the $-\mathrm{Au}-\mathrm{S}-\mathrm{Au}-\mathrm{S}$ - polymeric network and the absence of $\mathrm{Au}-\mathrm{Au}$ metallic bonds.

From TEM and XAS data we have obtained a very precise microstructural characterisation that allows us to explain the observed UV-Vis absorption behaviour of the samples. In general, the UV-Vis absorption spectra of gold nanoparticles are dominated in the $400-800 \mathrm{~nm}$ range by the presence of surface plasmon resonance 
bands. In our case, as the particles become smaller, a higher proportion of surface gold atoms bounded to sulphur lead to a localisation of charge at the Au-S bond that decreases the mobility of free electrons [22] and the intensity of absorption at the plasmon region. In fact, for the larger NPs in the Au-SR1 sample with a Au:S atomic ratio of 3.1, we still see the plasmon resonance in UV-Vis absorption spectra (see Fig.6). For the Au-SR2 sample, the plasmon resonance is not detectable for a $\mathrm{Au}: \mathrm{S}$ atomic ratio of 2.0. The $\mathrm{Au}(\mathrm{I})-\mathrm{SR}$ polymer sample shows a white colour and produces transparent solutions. In this case, the absence of metallic gold produces no light absorption in this wavelength region (see Fig.6). For the aged polymer sample, the formation of big gold particles hinders the solubility of the sample. In addition, the UVVis spectrum shows, together with a plasmon resonance feature at $570 \mathrm{~nm}$, a strong absorption along the wavelength range.

The effect of charge redistribution upon thiol-capped Au NPs formation and its size dependence were also examined with XPS. The Au $4 f$ core level for the Au-SR NPs and the polymer precursor material were measured for samples supported on polycarbonate filters. The results of this investigation are presented in Fig.7. In this figure, two important features previously investigated [24] are the target of new studies. First, the $\mathrm{Au} 4 f_{7 / 2}$ peak of the Polymer sample shifts (roughly $0.5 \mathrm{eV}$ ) to higher binding energy, as well as the $\mathrm{Au}_{2} \mathrm{~S}$ peak; this indicates that the $d$ charge at the Au site is depleted relative to the bulk gold. This shift is negligible when gold NPs are considered, due to their metallic character. Finally, a clear linewidth broadening is observed as the metallic character of the samples is lost. For the gold NPs and sulphide samples, the increase in the $\mathrm{Au} 4 f_{7 / 2}$ peak width is related to the fact that different amounts of $\mathrm{Au}-\mathrm{Au}$ bonds and $\mathrm{Au}-\mathrm{S}$ bonds are present in each sample; being $\mathrm{Au}_{2} \mathrm{~S}$ the sample with larger number of $\mathrm{Au}-\mathrm{S}$ bonds. The polymeric phase is highly unstable, therefore, many different Au phases coexist: $\mathrm{Au}-\mathrm{Au}, \mathrm{Au}-\mathrm{S}, \mathrm{Au}-\mathrm{radical}$ bonds and Au clusters. This would explain the significant linewidth broadening of the polymeric phase.

In addition, the $\mathrm{Au}$ valence band spectra were also measured. The results are shown in Fig.8. In this case, only bulk gold, Au-SR1, Au-SR2 and Polymer samples were considered. One interesting feature here is that the apparent spin-orbit splitting decreases as the metallic character is lost. The larger spin-orbit splitting corresponds to the bulk gold sample, whereas the rest of the samples from Au-SR1 to Polymer have a 
narrower spin-orbit splitting as the coordination number of the $\mathrm{Au}-\mathrm{Au}$ coordination sphere decreases.

In summary, three samples containing gold atoms functionalised with alkanethiol molecules (-SR) have been studied, all of them having different microstructures at the nanoscale. Two of them are gold NPs capped through Au-S bonds by the alkanethiol molecules and showing different particle sizes and different ratios of $\mathrm{Au}-\mathrm{S}$ to $\mathrm{Au}-\mathrm{Au}$ bonds. The polymeric-like sample contents however $100 \%$ of $\mathrm{Au}$ atoms bonded to sulphur in the alkanethiol molecules.

\section{Magnetic properties}

Fig.9 shows the magnetisation versus magnetic field curves measured at $5 \mathrm{~K}$ and room temperature for the two NPs, the $\mathrm{Au}(\mathrm{I})$-SR precursor polymer sample and the aged $\mathrm{Au}(\mathrm{I})-\mathrm{SR}$ polymer material. As we have previously reported [4], a clear ferromagnetic behaviour with a Curie temperature above $300 \mathrm{~K}$ is observed for the Au-SR NPs. Magnetisation values and observed coercive fields values are higher for the Au-SR2 sample $(0.095 \mathrm{emu} / \mathrm{g} \mathrm{Au}$ and $180 \mathrm{Oe})$ compared to the Au-SR1 sample $(0.005 \mathrm{emu} / \mathrm{g}$ $\mathrm{Au}$ and $115 \mathrm{Oe})$. As proposed in [4], the charge transfer from gold to sulphur and a high proportion of surface gold atoms as compared with bulk gold is necessary to generate a detectable permanent magnetism. In fact, only very small particles fulfil these requirements in such way that surface ferromagnetism can be detected. One must recall that surface ferromagnetism is overlapped to the diamagnetic behaviour of bulk gold. These considerations are in agreement with the observed behaviour of samples Au-SR1 and $\mathrm{Au}-\mathrm{SR} 2$, where a decrease in particle size and an increase in the S:Au ratio is promoting the presence of a ferromagnetic like behaviour. From this point of view, the polymer precursor $\mathrm{Au}(\mathrm{I})-\mathrm{SR}$ sample is showing a maximized $\mathrm{Au}$ to $\mathrm{S}$ charge transfer and a total dispersion of gold atoms so that $100 \%$ of them are bonded to sulphur. It is, however, shown that this is not a sufficient condition to show the permanent magnetism, histeresis and giant anisotropy. The data in Fig.9 shows that the simultaneous presence of $\mathrm{Au}-\mathrm{S}$ and $\mathrm{Au}-\mathrm{Au}$ bonds is necessary to observe these phenomena.

According to our first results [4], the permanent magnetism was explained as a consequence of blocking of the local magnetic moments (generated at the localised $\mathrm{Au}-$ $\mathrm{S}$ bonds by charge transfer) by giant magnetic anisotropies. However, the current results 
point out the orbital contribution to the observed magnetic behaviour as a consequence of the spin-orbit coupling so that localised charges and/or spins (Au-S bonds) can trap electrons (Au-Au bonds) in orbits [9]. In addition to that, it has been experimentally shown by XPS that spin-orbit coupling at the gold atom is decreasing significantly when the proportion of $\mathrm{Au}-\mathrm{S}$ bonds increases.

In the case of the aged polymer, the big gold nanoparticles have a small proportion of surface gold atoms bounded to sulphur so that the majority of gold atoms behave as bulk gold.

Regarding the Fe contamination always present in the samples, it is worth of mention that the level of $\mathrm{Fe}$ impurities cannot account for the observed magnetisation values in sample Au-SR2 even if all Fe is forming metallic Fe particles. In addition, we expect Fe impurities to be dispersed probably in an oxidised form. Besides, Dahr et al. [25] have recently shown that in the case of diluted magnetic semiconductors, a very low concentration of doping magnetic atoms can result in ferromagnetism at room temperature. Due to the fairly different electronic structure of semiconductors and metals it is not expected to find similar behaviour between semiconductors and gold nanoparticles.

\section{Conclusions}

Alkanethiolate-capped gold NPs have been obtained by a liquid-liquid phase reduction at room temperature based on the Brust method. The intermediate Au(I)-SR polymer phase obtained in the first step of the synthesis has been isolated together with two samples of NPs with different particle sizes and Au:S atomic ratios.

We have demonstrated that the polymeric phase contains gold atoms fully dispersed in an alkanethiol matrix with $100 \%$ of these Au atoms bonded to sulphur. The two NPs samples contain $\mathrm{Au}-\mathrm{Au}$ together with $\mathrm{Au}-\mathrm{S}$ bonds with increasing proportion of gold bond to sulphur for the particles of smaller size.

It has been experimentally demonstrated that the simultaneous presence of $\mathrm{Au}-\mathrm{Au}$ and $\mathrm{Au}-\mathrm{S}$ bonds is necessary to detect a ferromagnetic behaviour in thiol derivatised gold 
nanostructures. It is proposed that the orbital momentum induced at the surface conduction electrons is crucial to understand the observed giant anisotropy. The orbital motion is driven by localised charge and/or spin through spin-orbit interactions, that reaches extremely high values at the surface.

\section{Acknowledgements}

XAS facilities at BM29 in ESRF and the technical support from G.L. Ciatto are acknowledged. Financial support from the Spanish MEC (NAN2004-09125-C07) and "Junta de Andalucía" is also acknowledged.

\section{References}

[1] Sampedro B, Crespo P, Hernando A, Litrán R, Sánchez-López JC, López-Cartes C, Fernández A, Ramírez J, González-Calbet J, Vallet M. Phys Rev Lett 2003;91:237203.

[2] Shinoara T, Sato T, Taniyama T. Phys Rev Lett 2003;91:197201.

[3] Carmeli I, Leitus G, Naaman R, Reich S, Vager Z. J Chem Phys 2003;118:10372.

[4] Crespo P, Litrán R, Rojas TC, Multigner M, de la Fuente JM, Sánchez-López JC, García MA, Hernando A, Penadés S, Fernández A. Phys Rev Lett 2004;93:087204.

[5] Litrán R, Sampedro B, Rojas TC, Multigner M, Sánchez-López JC, Crespo P, López-Cartes C, García MA, Hernando A, Fernández A. Phys Rev B 2006;73:054404.

[6] Kumar V, Kawazoe Y. Eur Phys J D 2003;24:81.

[7] Hernando A, Sampedro B, Litrán R, Rojas TC, Sánchez-López JC, Fernández A. Nanotechnology 2006;17:1449.

[8] Vager Z, Naaman R. Phys Rev Lett 2004;92:087205.

[9] Hernando A, Crespo P, García MA. Phys Rev Lett 2006;96:057206.

[10] Fernández A, Guerrero E, Rojas TC, Multigner M, Crespo P, Hernando A. Phys Rev Lett; submitted.

[11] Hernando A, Crespo P, García MA, Fernández Pinel E, Fernández A, Penadés S. Phys Rev Lett; submitted.

[12] Brust M, Walter M, Bethell D, Schiffrin DJ, Whyman R. J Chem Soc Chem Commun 1994;801.

[13] Chen S, Templeton AC, Murray RW. Langmuir 2000;16:3543.

[14] Templeton AC, Wuelfin WP, Murray RW. Acc Chem Res 2000;33:27. 
[15] Hostetler MJ, Wingate JE, Zhong Ch-J, Harris JE, Vachet RW, Clark MR, Londono JD, Green SJ, Stokes JJ, Wignall GD, Glish GL, Porter MD, Evans ND, Murray RW. Langmuir 1998;14:17.

[16] Bonin D, Kaiser P, Freitigny C, Desbarres J. Structures fines d'absorption des rayons $\mathrm{X}$ en chimie, 3. Logiciels d'analyse EXAFS. In: Dexpert H, Michalowizc A, Verdagner M, editors. Societé Française de Chimie: Paris, 1989.

[17] Zabinsky SI, Rehr JJ, Ankudinov A, Albers RC, Eller MJ. Phys Rev B $1995 ; 52: 2995$.

[18] Shirley DA. Phys Rev B 1972;5:4709.

[19] Yeh JJ, Lindau I. Atom Dat and NucDat Tab 1985;32:1.

[20] XPSPEAK v. 4.1: Available at http://www.phy.cuhk.edu.hk/ surface/XPSPEAK/.

[21] López-Cartes C, Rojas TC, Litrán R, Martínez-Martínez D, de la Fuente JM, Penadés S, Fernández A. J Phys Chem B 2005;109:8761.

[22] García MA, de la Venta J, Crespo P, LLopis JJ, Penadés S, Fernández A, Hernando A. Phys Rev B 2005;72:241403(R).

[23] Zhang P, Sham TK. Appl Phys Lett 2002;81:736.

[24] Zhang P, Sham TK. Phys Rev Lett 2003;90:245502.

[25] Dhar S, Brandt O, Ramsteiner M, Sapega VF, Ploog KH. Phys. Rev. Lett. 2005;94:037205. 


\section{Figures}
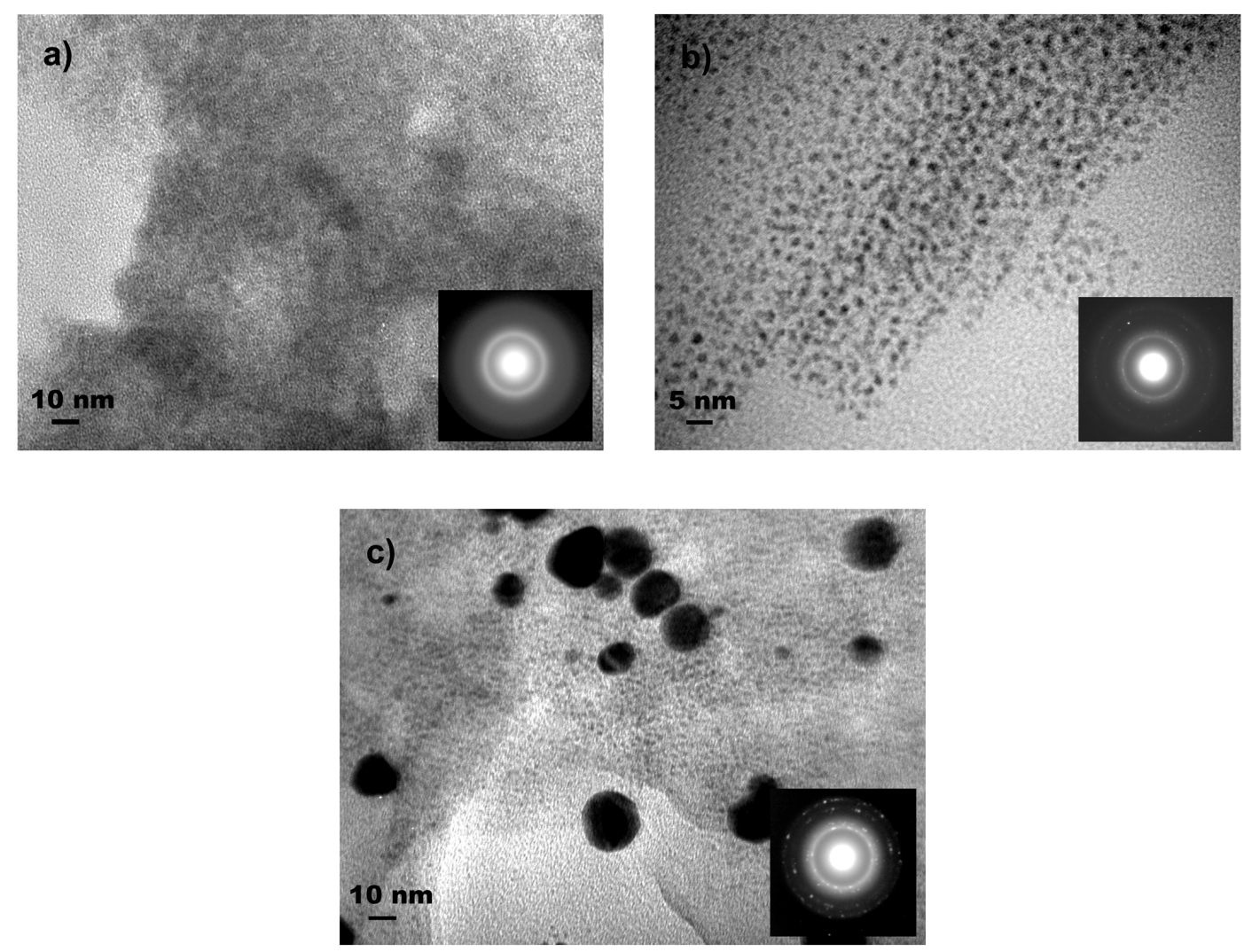

Figure 1. TEM micrograph and the corresponding selected area electron diffraction (SAED) patterns for the following samples: a) Polymer, b) Polymer under e-beam irradiation and c) Polymer-aged.
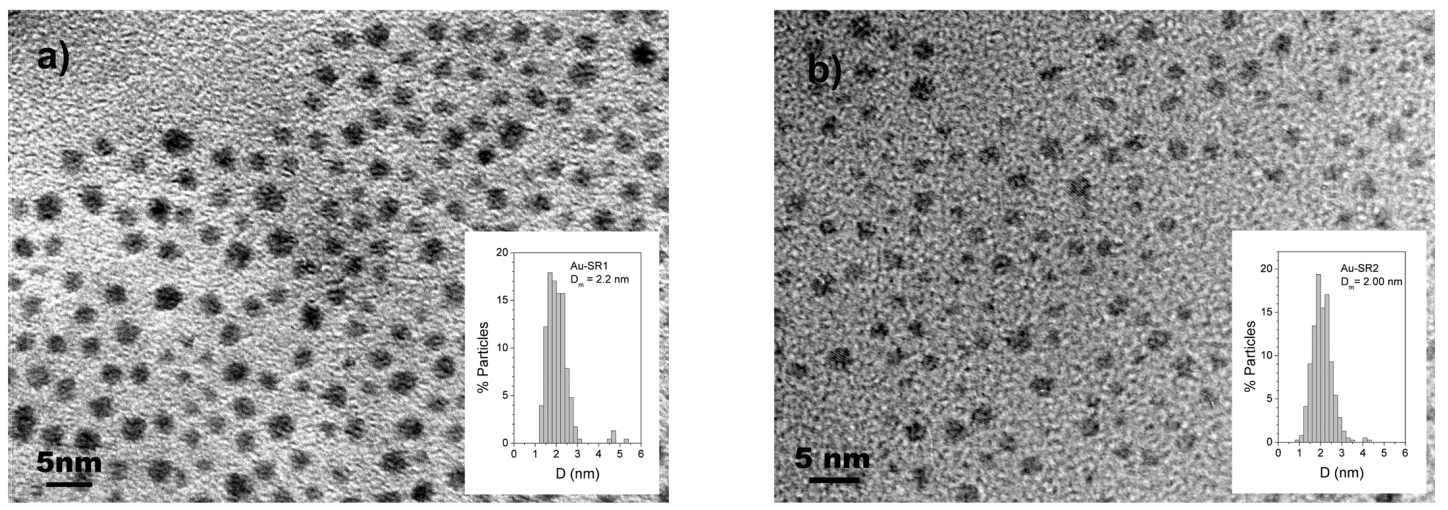

Figure 2. TEM micrograph and particle size distribution histogram of the Au-SR1 (a) and Au-SR2 (b) NPs. 


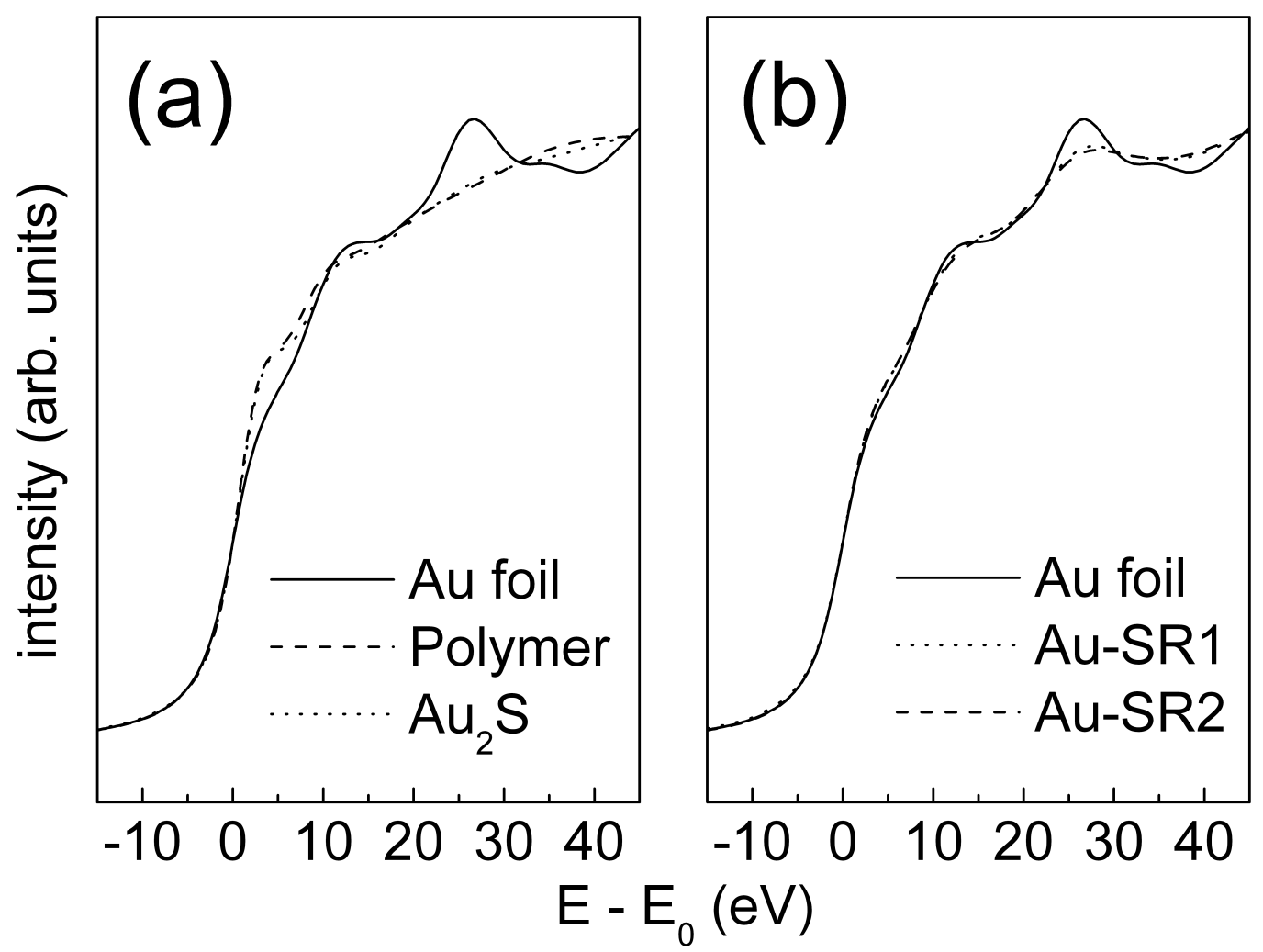

Figure 3. Au L2-XANES spectra for the different samples compared to bulk gold (Au foil): a) The intermediate polymeric precursor and $\mathrm{Au}_{2} \mathrm{~S}$ reference sample; b) Au-SR1 and Au-SR2 samples. 


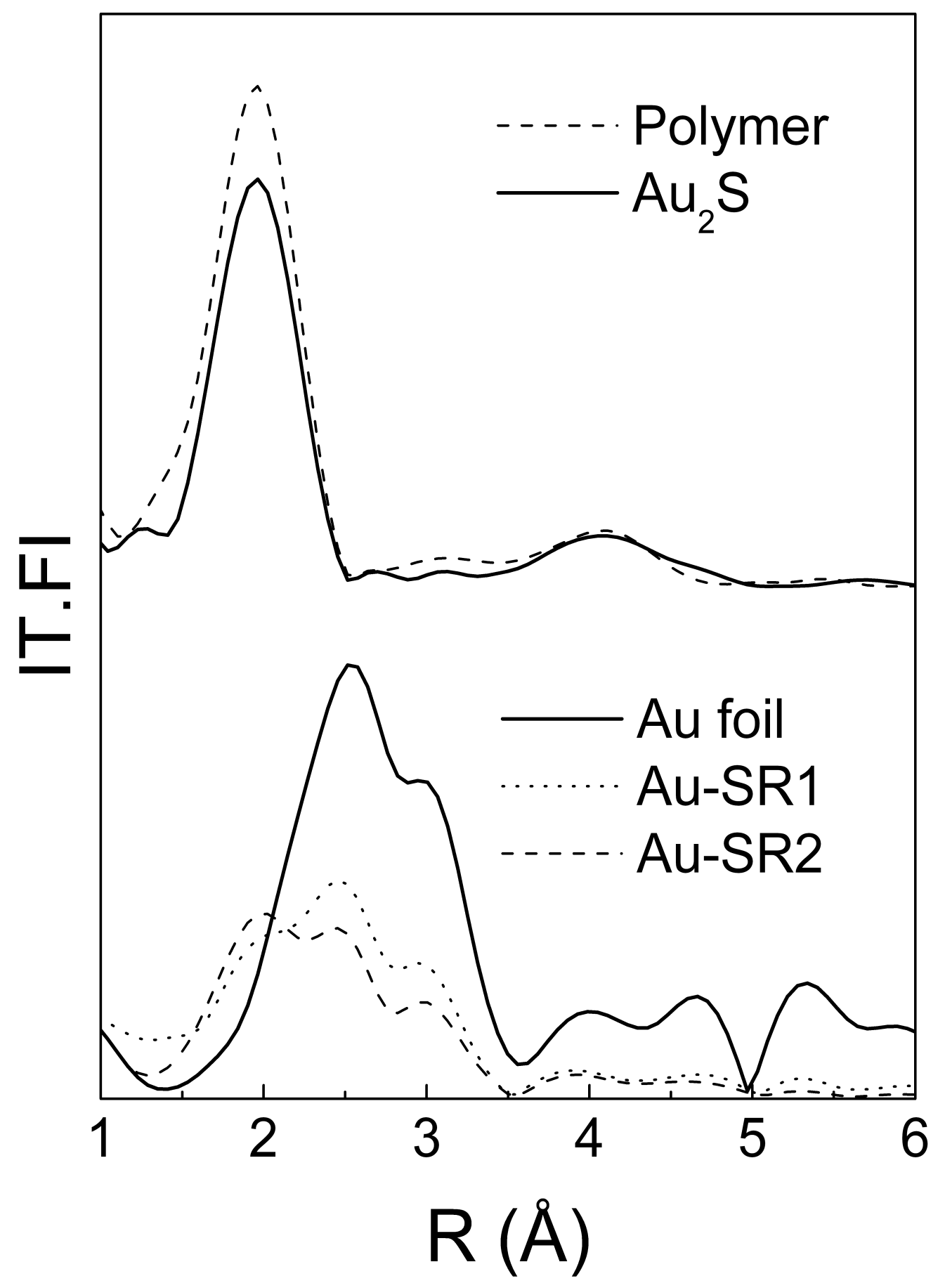

Figure 4. Modulus of the Fourier Transform of EXAFS oscillations at the Au L3-edge (k weighted, k space range of 2.8-13.2 $\AA$ without phase corrections) for the Au-SR2, $\mathrm{Au}-\mathrm{SR} 1$ and Polymer samples compared to bulk gold ( $\mathrm{Au}$ foil) and $\mathrm{Au}_{2} \mathrm{~S}$ reference samples. 

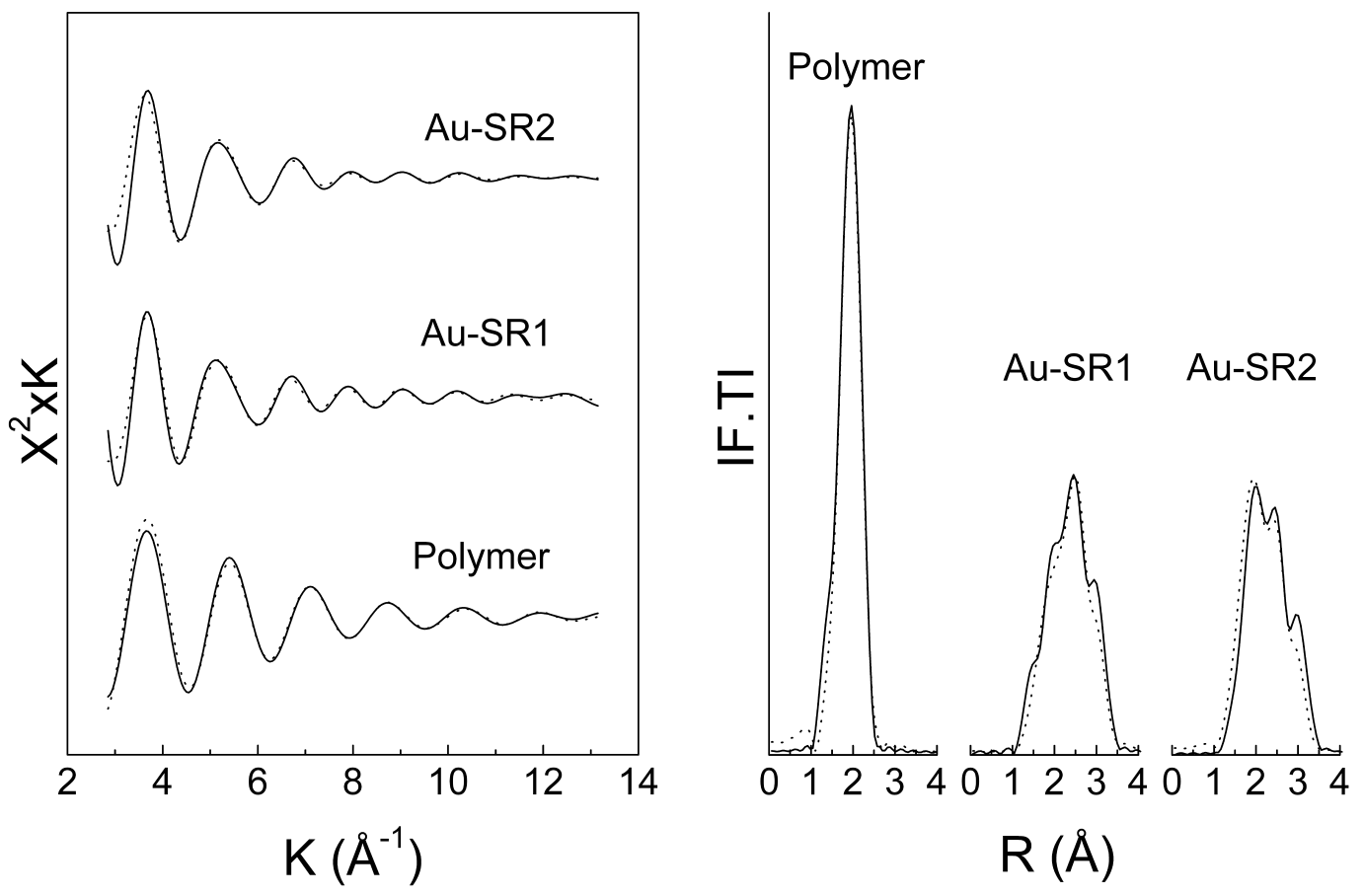

Figure 5. Experimental (-) and fitted (…) EXAFS oscillations and radial distribution functions for Au-SR1, Au-SR2 and Polymer samples. 


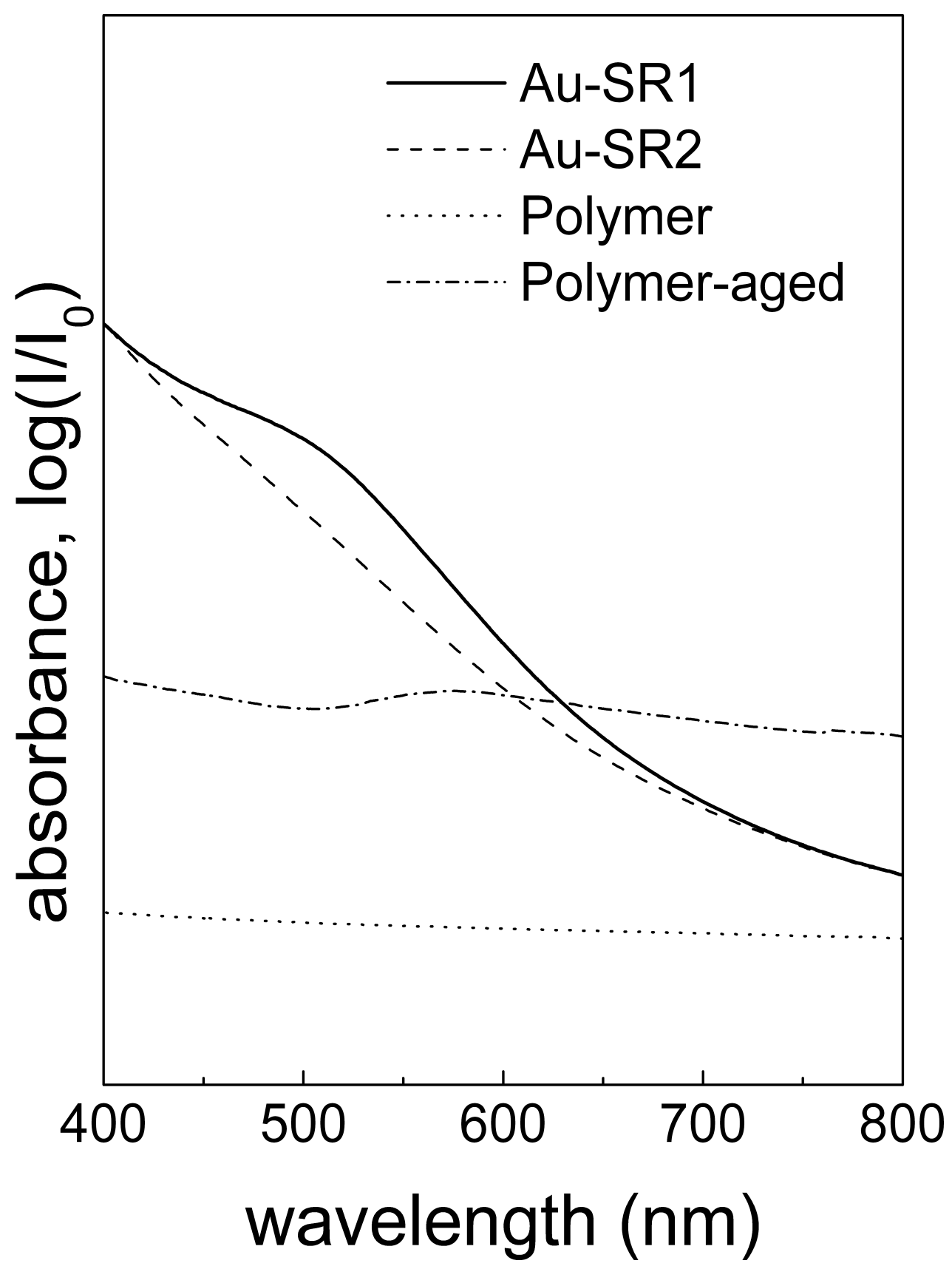

Figure 6. UV-Visible absorption spectra for the Au-SR capped nanoparticles and the intermediate polymeric phase. 


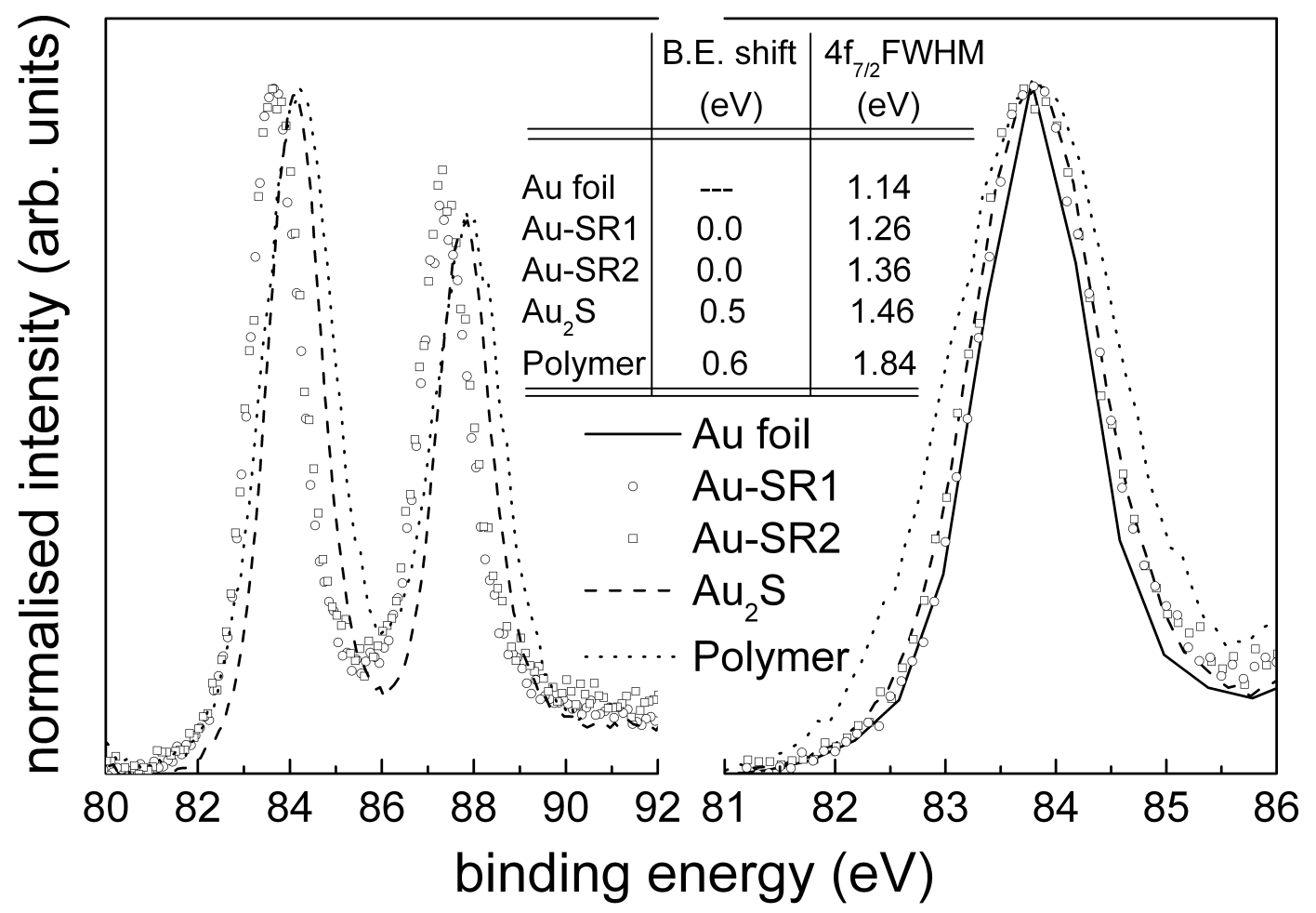

Figure 7 Energy shift of the Au $4 \mathrm{f}$ XPS peaks (on the left) for the Au-SR1, Au-SR2, $\mathrm{Au}_{2} \mathrm{~S}$, and intermediate polymeric phase. On the right, the $\mathrm{Au} 4 \mathrm{f}_{7 / 2}$ XPS peak broadening is shown for the gold bulk, $\mathrm{Au}-\mathrm{SR} 1, \mathrm{Au}-\mathrm{SR} 2, \mathrm{Au}_{2} \mathrm{~S}$, and intermediate polymeric phase samples.

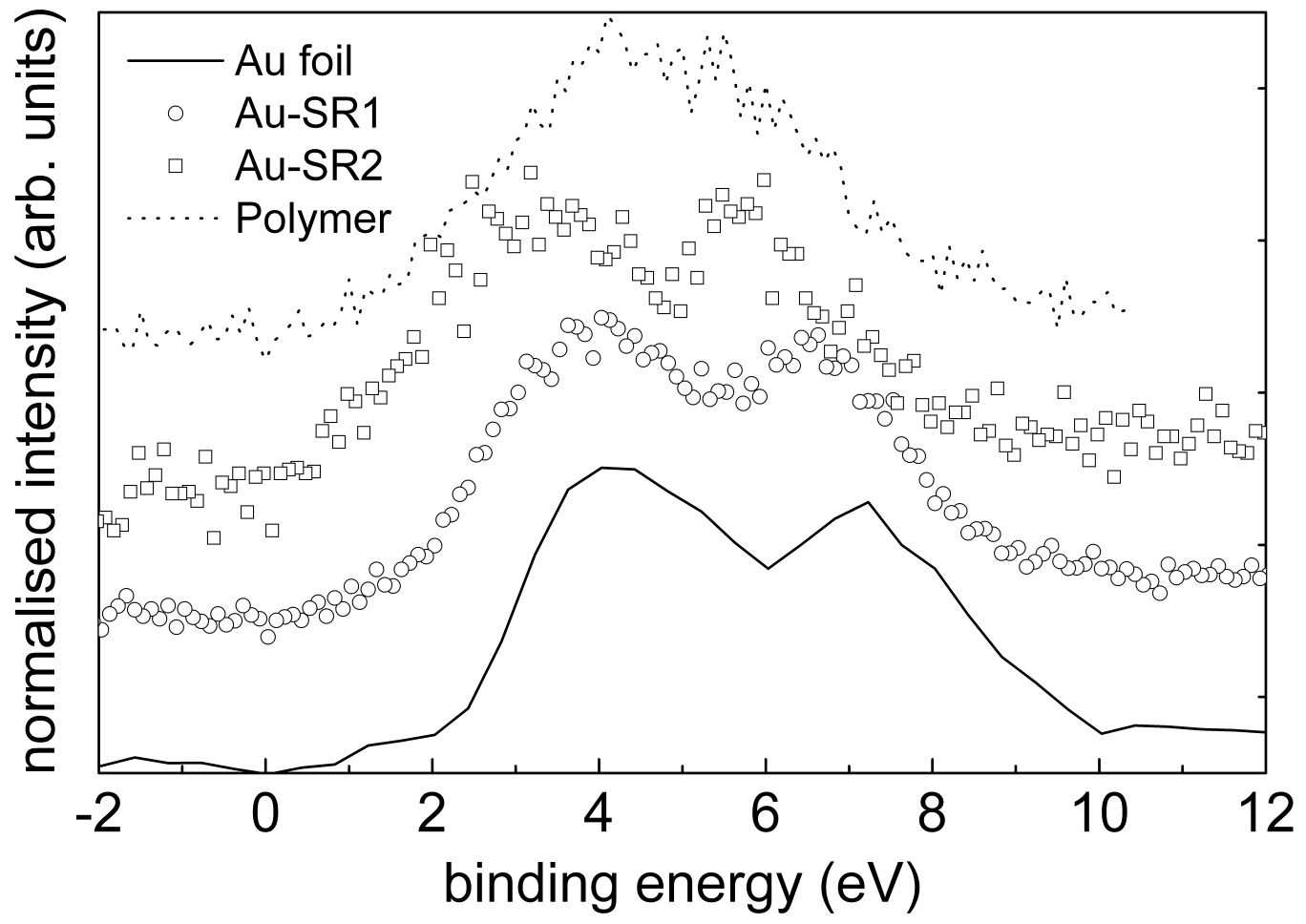

Figure 8. Au valence band ( $5 \mathrm{~d}$ and $6 \mathrm{~s}$ levels) XPS spectra for the bulk gold, Au-SR1, $\mathrm{Au}-\mathrm{SR} 2$ and polymer samples. 

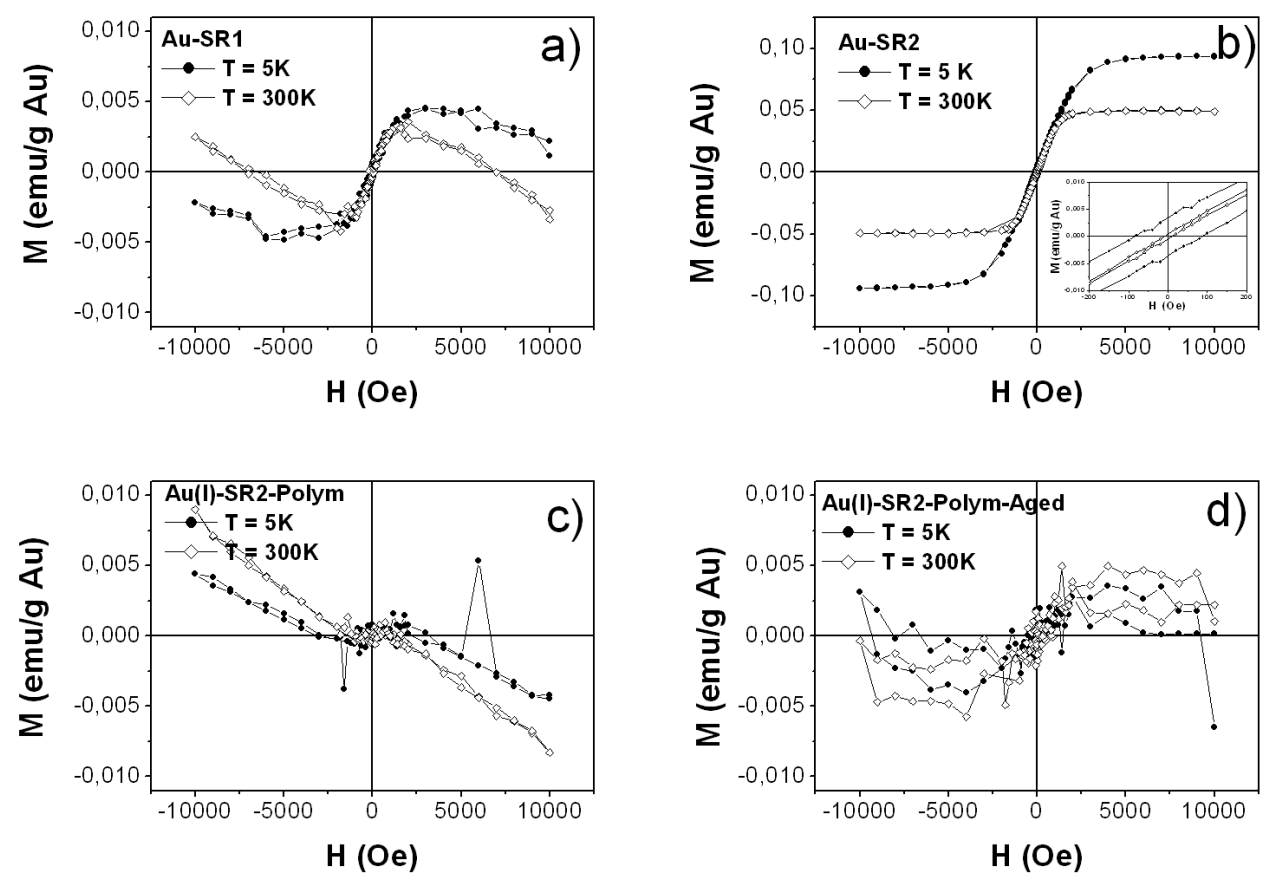

Figure 9. Hysteresis loops corresponding to the gold thiol-capped nanoparticles AuSR1 (a), Au-SR2 (b), the intermediate polymer precursor (c) and the aged polymer (d), at 5 and $300 \mathrm{~K}$. 
Table 1. Size and chemical composition data for the selected samples.

\begin{tabular}{|c|c|c|c|c|c|c|c|c|c|}
\hline Sample & $\begin{array}{c}\text { Au } \\
\text { wt\% }\end{array}$ & $\begin{array}{c}\text { Fe } \\
\text { wt } \%\end{array}$ & $\begin{array}{c}\text { S } \\
w t \%\end{array}$ & $\begin{array}{c}\mathrm{C} \\
\mathrm{wt} \%\end{array}$ & $\begin{array}{c}\text { H } \\
\text { wt } \%\end{array}$ & $\begin{array}{c}\mathrm{Au} / \mathrm{S} \\
\text { (at. ratio) }\end{array}$ & $\begin{array}{c}\text { Dm } \\
(\mathbf{n m}) \\
\text { (a) }\end{array}$ & $\begin{array}{c}\text { Total } \\
\text { Nr } \\
\text { (b) }\end{array}$ & $\begin{array}{c}\% \mathrm{Nr} \\
\text { (c) }\end{array}$ \\
\hline Au-SR1 & 70.5 & 0.018 & 3.7 & 23.6 & 4.1 & $\begin{array}{l}3.1 \\
3.0^{(\mathrm{d})}\end{array}$ & 2.2 & 249 & 66.7 \\
\hline Au-SR2 & 50.3 & 0.007 & 4.1 & 24.3 & 4.4 & $\begin{array}{l}2.0 \\
2.4^{(\mathrm{d})}\end{array}$ & 2.0 & 177 & 68.9 \\
\hline Polymer & 49.5 & 0.012 & 7.8 & 36.4 & 6.2 & $\begin{array}{l}1.0 \\
1.0^{(\mathrm{d})}\end{array}$ & --- & --- & --- \\
\hline
\end{tabular}

(a) Dm: Average particle size as calculated from particle size distribution histograms.

(b) Total Nr: Calculated number of atoms for a pure gold cluster.

(c) \%Nr: Calculated percentage of atoms located at the surface of a pure gold cluster.

(d) Atomic ratio determined from XPS. 
Table 2. Best fitting parameters of the Au L3-edge EXAFS oscillations of samples.

\begin{tabular}{|c|c|c|c|c|c|}
\hline Sample & (a) & $\begin{array}{l}\mathbf{N} \\
\text { (b) }\end{array}$ & $\begin{array}{c}\mathbf{R}(\mathbf{A}) \\
(\mathbf{c}) \\
( \pm 0.02 \AA \dot{A})\end{array}$ & 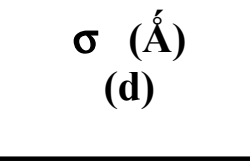 & $\begin{array}{c}\Delta \sigma^{2} \times 10^{-3} \\
\left(\AA^{2}\right)\end{array}$ \\
\hline Au foil & $(\mathrm{Au}-\mathrm{Au})_{\mathrm{m}}$ & 12 & 2.85 & $0.073 \pm 0.015$ & \\
\hline Au-SR1 & $\begin{array}{c}(\mathrm{Au}-\mathrm{S}) \\
(\mathrm{Au}-\mathrm{Au})_{\mathrm{m}}\end{array}$ & $\begin{array}{c}0.45 \pm 0.09 \\
8.8 \pm 1.8\end{array}$ & $\begin{array}{l}2.30 \\
2.80\end{array}$ & $\begin{array}{l}0.090 \pm 0.018 \\
0.094 \pm 0.019\end{array}$ & 3.5 \\
\hline Au-SR2 & $\begin{array}{c}(\mathrm{Au}-\mathrm{S}) \\
(\mathrm{Au}-\mathrm{Au})_{\mathrm{m}}\end{array}$ & $\begin{aligned} 0.71 & \pm 0.14 \\
6.8 & \pm 1.4\end{aligned}$ & $\begin{array}{l}2.29 \\
2.77\end{array}$ & $\begin{aligned} 0.091 & \pm 0.018 \\
0.10 & \pm 0.02\end{aligned}$ & 4.5 \\
\hline Polymer & $(\mathrm{Au}-\mathrm{S})$ & $1.3 \pm 0.3$ & 2.28 & $0.036 \pm 0.007$ & \\
\hline $\mathbf{A u} \mathbf{u}_{2} \mathbf{S}$ & $(\mathrm{Au}-\mathrm{S})$ & $1.7 \pm 0.3$ & 2.31 & $0.063 \pm 0.013$ & \\
\hline
\end{tabular}
(a) Type of atoms in the coordination shell.
(b) $\mathrm{N}$ : coordination number.
(c) R: bond length.
(d) $\sigma$ : Debye-Waller factor. 